\title{
Effect of a Steady Flow and an Atmospheric Magnetic Field on the Solar $p$ - and $f$-Modes
}

\author{
R. Erdélyi and Y. Taroyan \\ Department of Applied Mathematics, University of Sheffield, Hicks \\ Building, Hounsfield Road, Sheffield S3 7RH, UK. \\ Email: Robertus@sheffield.ac.uk
}

\begin{abstract}
The combined effect of a subsurface steady homogeneous flow and a chromospheric uniform magnetic field on the solar $p$ - and $f$-modes is evaluated theoretically for a simple model of the Sun. The derived dispersion relation is solved analytically in limit of the long wavelength approximation and is evaluated numerically for arbitrary wavelengths. The influence of an equilibrium flow is more dominant in limit of small wavenumbers. For arbitrary wavelengths the effect of a magnetic field might be stronger than frequency shifts caused by a steady homogeneous flow.
\end{abstract}

\section{Introduction}

The aim of our study is to evaluate theoretically the combined effect of large-scale sub-surface motions (e.g., a meridional flow) and an atmospheric magnetic field on the frequencies of the solar $p$ - and $f$-modes. Erdélyi, R., Varga, E., \& Zétényi M. (1999) have studied the effect of a sub-surface motion on magnetoacousticgravity surface waves in a model of the solar interior - solar atmosphere interface. The main characteristics of their isothermal atmosphere was a magnetic though constant- $\beta$ plasma, while in the sub-surface interior region there was a uniform and homogeneous equilibrium flow. They found the flow causes a shift of the MAG modes, which in certain cases bifurcate. Erdélyi \& Taroyan (1999) generalised the above model by allowing the temperature to increase linearly with depth in the sub-surface zone. They derived the dispersion relation and analytical formulae for the frequencies of $p$ - and $f$-modes in the limit of small wavenumbers. Numerical solutions were presented for other cases.

In the present paper, we introduce a horizontal uniform flow $\mathbf{u}_{\mathbf{0}}=(V, 0,0)$ in the lower (internal) region $z>0$, while the upper atmospheric region of the model is embedded in a uniform magnetic field. This solar model, without a flow has been considered by, e.g., Evans \& Roberts (1990). We derive the dispersion relation and solve it analytically for the small wavenumber case. The obtained dispersion relation is also evaluated numerically for arbitrary wavelengths. 


\section{The small wavenumber approximation}

Using the notations of Evans \& Roberts (1990), we derive the following formulae for frequency shifts from the obtained dispersion relation (which contains hypergeometric and confluent hypergeometric functions) in the limit of small wavenumbers, i.e., when $K \rightarrow 0$ :

$$
\begin{array}{r}
\Omega=\Omega_{n}+\frac{V}{c_{s p}} \sqrt{\frac{\gamma_{p}}{2(m+1)}}(2 K)^{\frac{1}{2}}+\frac{\Gamma(1+m+n) \gamma_{c}\left(1-\Omega_{n}^{4}-2 \Omega_{n}^{2}\right)}{\Gamma(1+m) \Gamma(2+m) \Gamma(n)\left(\gamma_{c}+2 \beta\right) \Omega_{n}\left(1-\Omega_{n}^{4}\right)} \\
\times\left[\frac{m+1}{\gamma_{p}}+\left(\frac{m+1}{\gamma_{p}}-m\right)\right]^{-1}(2 K)^{m+1}
\end{array}
$$

for $n=1,2, \ldots p$-modes and

$$
\Omega=1+\frac{V}{c_{s p}} \sqrt{\frac{\gamma_{p}}{2(m+1)}}(2 K)^{\frac{1}{2}}+\frac{\gamma_{c}}{2 \Gamma(m+2)\left(2 \beta+\gamma_{c}\right)}(2 K)^{m+1}
$$

for the $f$-mode.

Studying these formulae we see that in the limit of small wavenumbers the flow has a stronger influence on the $p$ - and $f$-mode frequencies than the atmospheric magnetic field.

\section{Numerical Results}

Numerical results are obtained and presented in Fig. 1 by solving the full dispersion relation and taking the same parameter values as in Evans \& Roberts (1990). In the presence of a uniform magnetic field there are no cutoff frequencies, unlike in the case with no magnetic field.

Recent measurements of $p$-mode frequencies (Braun \& Fan 1998) show a significant frequency shift between poleward- and equatorward-traveling waves measured over solar latitudes $20^{\circ}-60^{\circ}$, which is consistent with the Doppler effect of a poleward meridional flow of the order of $10 \mathrm{~m} / \mathrm{s}$. The measurements show frequency shifts in the range of, e.g., $1-3 \mu \mathrm{Hz}$ for $l=477$. As one can see from, e.g., our last figure, the frequency shift predicted by our model is in agreement with these measurements.

Acknowledgments. The authors thank B. Roberts and K. Murawski for stimulating discussions and their comments. RE acknowledges M. Kéray for patient encouragement. RE also acknowledges the financial support obtained from the NSF, Hungary (OTKA, ref nr. TO32462). YT is grateful to the White Rose Consortium and the Symposium organisers for their financial support.

\section{References}

Erdélyi, R., Varga, E., \& Zétényi, M. 1999, in ESA-SP-448, 1, 269

Erdélyi, R., \& Taroyan, Y. 1999, in ESA-SP-448, 1, 81

Evans, D. J., \& Roberts, B. 1990, ApJ, 356, 704

Braun, D. C., \& Fan, Y. 1998, ApJ, 508, L105 

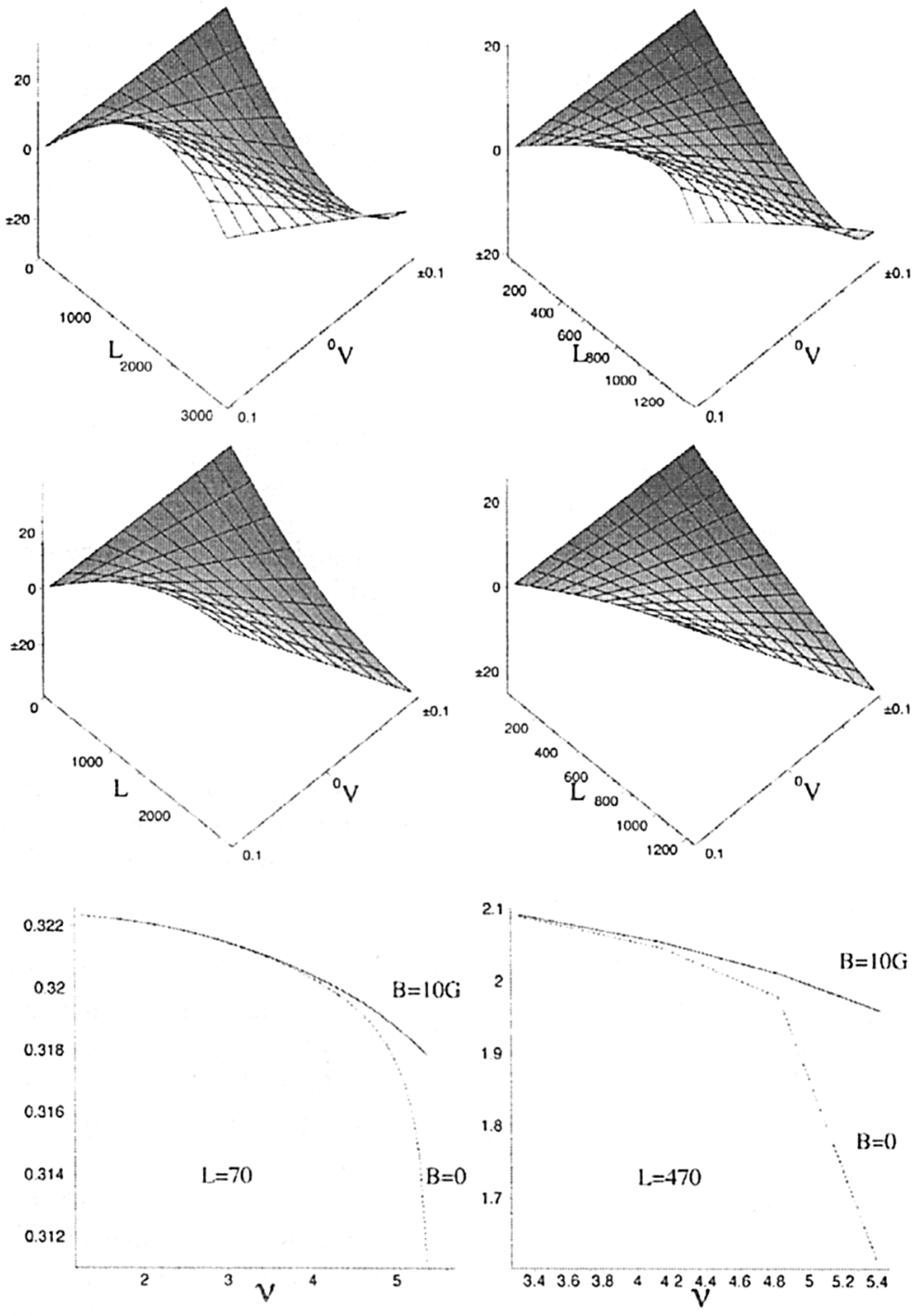

Figure 1. Cyclic frequency shift $\Delta_{V} \nu=\nu(l, B, V)-\nu(l, B, 0)(\mu \mathrm{Hz})$ with $B=0$ (upper panels) and $B=30 \mathrm{G}$ (middle panels) as a function of the spherical harmonic degree $l$ and the flow $V(\mathrm{~km} / \mathrm{s})$ for the $f$ - and $n=1 p$-mode; $\Delta_{ \pm V} \nu=\nu(l, B, V)-\nu(l, B,-V)(\mu \mathrm{Hz})$ with $V=10$ $\mathrm{m} / \mathrm{s}$ as a function of the frequency $\nu(l, B, 0)$ (lower panels) for $p$-modes. 